\title{
Fentanyl induces autophagy via activation of the ROS/MAPK pathway and reduces the sensitivity of cisplatin in lung cancer cells
}

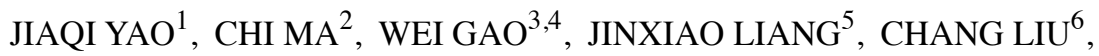 \\ HONGFANG YANG $^{1}$, QIU YAN ${ }^{3}$ and QINGPING WEN ${ }^{1}$
}

\begin{abstract}
Departments of ${ }^{1}$ Anesthesiology and ${ }^{2}$ Surgery, The First Affiliated Hospital of Dalian Medical University;
${ }^{3}$ Department of Biochemistry and Molecular Biology, Dalian Medical University, Liaoning Provincial Core Laboratory of Glycobiology and Glycoengineering, Dalian, Liaoning 116011; ${ }^{4}$ Department of Clinical Medicine, Zhejiang University

City College School of Medicine, Hangzhou, Zhejiang 310015; ${ }^{5}$ Department of Surgery, Zhejiang Cancer Hospital, Hangzhou, Zhejiang 310012; ${ }^{6}$ Department of Anesthesiology, Peking University Third Hospital, Beijing 100191, P.R. China
\end{abstract}

Received March 18, 2016; Accepted August 8, 2016

DOI: $10.3892 /$ or.2016.5183

\begin{abstract}
Cancer pain is the most common complication of lung carcinoma. Opioid agonist fentanyl is widely used for relieving pain in cancer patients, and cisplatin (DDP)-based chemotherapy is commonly used for the treatment of advanced lung cancer; these two drugs are always used together in lung carcinoma patients. However, the mechanisms and related biological pathways by which fentanyl influences cisplatin sensitivity are relatively poorly reported. Here, we found that fentanyl reduces the sensitivity of cisplatin in human lung cancer cells and induces autophagy. Fentanyl induced reactive oxygen species (ROS) generation and JNK activation. N-acetyl-L-cysteine is a ROS scavenger and antioxidant, and the inhibition of JNK with SP600125 prevented fentanyl-induced autophagy. We also found that 3-methyladenine (3-MA; an autophagy inhibitor) increased the sensitivity of DDP and weakened the inhibition of fentanyl. In conclusion, fentanyl reduces the sensitivity of cisplatin in lung cancer cells through the ROS-JNKautophagy pathway, whereas the autophagy inhibitor 3-MA may weaken this effect.
\end{abstract}

Correspondence to: Professor Qiu Yan, Department of Biochemistry and Molecular Biology, Dalian Medical University, Liaoning Provincial Core Laboratory of Glycobiology and Glycoengineering, Dalian, Liaoning 116044, P.R. China

E-mail: yanqiu63@126.com

Professor Qingping Wen, Department of Anesthesiology, The First Affiliated Hospital of Dalian Medical University, 222 Zhongshan Road, Dalian, Liaoning 116011, P.R. China

E-mail:dmuwqp@163.com

Key words: fentanyl, lung cancer, autophagy, cisplatin, reactive oxygen species, JNK

\section{Introduction}

Lung cancer is the most common cause of cancer-related death in males and second most common cause in females (1). Recently, the morbidity and mortality of lung carcinoma has noticeably increased. Lung cancer is classified as non-small cell lung cancer (NSCLC) and small cell lung cancer (SCLC). NSCLC represents approximately $85 \%$ (2) of cases and has a five-year survival of only $15 \%$ (3).

Pain relief is a fundamental and formidable task in the treatment of cancer patients because most cancer patients have severe pain (4). The $\mu$-opioid receptor was proposed as the third step on the analgesic ladder and is used to relieve pain for terminal cancer patients according to WHO guidelines.

Fentanyl is widely used for relieving pain and narcotizin. Specifically, it is considered a good analgesic that is effective against cancer pain in terminal cancer patients (5). Recently, the effects of opioid analgesics on cancer patients have gained increasing attention. Basic research indicates that opioids induce tumour growth, inhibit apoptosis, and promote angiogenesis. However, the effect of fentanyl on autophagy has not been reported. We found that fentanyl increases autophagy in lung cancer cells. Autophagy is an evolutionarily conserved catabolic process that involves the degradation of cellular components through the lysosomal machinery (6). Autophagy may remove and assist long-standing proteins in metabolic waste recycling to maintain a stable cell environment. A recent study reported that autophagy exerts a protective effect when cells experience increased pressure and contributes to cellular survival by providing nutrients and energy to help cells adapt to starvation or stress (such as hypoxia, X-rays and anticancer drugs) (7).

LC3 and Beclin-1 are two key proteins involved in autophagy. In this study, we demonstrate that fentanyl induces higher levels of LC3-II. LC3-II formation is regarded as reliable biochemical evidence of autophagy as the amount of LC3-II usually correlates well with the number of autophagosomes. In our study, Beclin-1 expression also increased in a dose-dependent manner. Beclin-1 plays a role in the initiation step and is essential for autophagosome formation (8). In a 
previous study, the epidermal growth factor receptor (EGFR) inhibitors gefitinib and erlotinib both induced autophagy in lung cancer cells, and hypoxia-induced autophagy mediates cisplatin resistance in lung cancer cells (9). Therefore, in the present study, we investigated whether fentanyl, which is commonly used, alters sensitivity to cisplatin in the human lung cancer cell line A549.

Chemotherapy is the main method of treating lung cancer in the early stages of disease, particularly as postoperative adjuvant chemotherapy. Platinum drugs are the most common chemotherapy agents used to treat lung cancer. Combining cisplatin (DDP) and other chemotherapy drugs for middle-late lung cancer patients has become a standardized treatment. Although the rate of survival has improved, sensitivity has decreased, which is a major obstacle in the clinical application of chemotherapy. The direct effects of opioids on cancer cell sensitivity to chemotherapy have not been adequately examined. Therefore, in the present study, we investigated whether fentanyl, which is commonly used during general anaesthesia, alters sensitivity to cancer chemotherapy in a human lung cancer cell line (10).

Increasing evidence indicates that multiple stress conditions, such as oxidative stress (11), endoplasmic reticulum (ER) stress and pathogen infection (12), induce autophagy through different molecular pathways. Among them, reactive oxygen species (ROS) function as signalling molecules not only in cell growth, differentiation, proliferation and apoptosis (13) but also in autophagy (14). ROS are active forms of oxygen generated as by-products from cellular metabolism. Furthermore, ROS affect various signalling pathways, such as the MAPK (including ERK, JNK and p38) signal transduction cascades. Recently, it was demonstrated that p38 MAPK mediates autophagy in response to chemotherapeutic agents (15). JNK, also known as the stress-activated protein kinase, has been implicated in apoptosis and autophagy (15-17). In mammals, there are three JNK genes: JNK1, JNK2 and JNK3 (18). JNK1 and JNK2 are ubiquitously expressed, whereas JNK3 is primarily expressed in the brain, cardiac smooth muscle and testes (18). In particular, ROS induces JNK-dependent autophagy $(19,20)$.

Our data demonstrated that fentanyl, via the activation of ROS-JNK-mediated autophagy, inhibits the chemotherapeutic sensitivity of DDP. The autophagy inhibitor 3-methyladenine (3-MA) may weaken this effect.

\section{Materials and methods}

Reagents and antibodies. Fentanyl hydrochloride and DDP was obtained from Northeast Pharmaceutical Group Co., Ltd., Shenyang, China). N-Acetyl-L-cysteine (NAC) were purchased from Beyotime Institute of Biotechnology (Shanghai, China), JNK inhibitor (SP600125), and 3-MA were purchased from Selleck Chemicals (Houston, TX, USA).

Cell culture. The human NSCLC cell lines A549 and SPC-A1 were obtained from the Shanghai Institute of Biochemistry and Cell Biology, Chinese Academy of Sciences (Shanghai, China) and maintained in Dulbecco's modified Eagle's medium (DMEM) supplemented with $10 \%$ fetal bovine serum and 1\% penicillin/streptomycin (all from Gibco-BRL,
Invitrogen Life Technologies, Gaithersburg, MD, USA) in a humidified cell culture incubator at $37^{\circ} \mathrm{C}$ and in $5 \% \mathrm{CO}_{2}$.

Cell viability assay. The cells $\left(3 \times 10^{3} /\right.$ well) were seeded in 96-well plates and incubated overnight. After treatment, $10 \mu \mathrm{l}$ of the Cell Counting Kit-8 (CCK-8) reagent (Dojindo Molecular Technologies, Inc., Kumamoto, Japan) was added to $90 \mu \mathrm{l}$ of media in each well, followed by a 2 -h incubation, after which the absorbance was measured at $450 \mathrm{~nm}$ using a microplate reader (Thermo Fisher Scientific, Waltham, MA, USA). Each assay was conducted in quadruplicate and repeated at least three times.

Brightfield images. Cell monolayers were cultured for $24 \mathrm{~h}$ in 2-well glass-covered chamber slides and then treated with various drugs. After washing with PBS, micrographs were taken using an Olympus inverted fluorescence microscope (Olympus, Tokyo, Japan). The number of cells with increased acidic vesicular organelles was determined by counting at least three representative fields per treatment condition, and a minimum of three replicate experiments was conducted.

Apoptosis assay. Apoptosis was assessed by Annexin V-binding analysis of flow cytometry. For flow cytometric analysis, cells $\left(1 \times 10^{5}\right)$ were seeded in $6-\mathrm{cm}$ dishes overnight before treatment. Both adherent and floating cells were harvested and combined, washed twice with PBS, resuspended in $500 \mu \mathrm{l}$ of binding buffer, and stained using an Annexin V-FITC/PI kit (Nanjing KeyGen Biotech Co., Ltd., Nanjing, China) according to the manufacturer's instructions. After incubation in the dark for $30 \mathrm{~min}$, the cells were analyzed using FACSCalibur instrument (FACSCalibur; BD Biosciences, San Jose, CA, USA). All experiments were performed in duplicate with reproducibility.

DAPI staining. Cells were grown on glass coverslips. After washing with PBS, cells were fixed in $4 \%$ paraformaldehyde-PBS for $30 \mathrm{~min}$ and treatment followed with $0.1 \%$ Triton X-100 for $10 \mathrm{~min}$ at $4^{\circ} \mathrm{C}$. Then stained with DAPI (Beyotime Institute of Biotechnology), washed with PBS. Images were captured with the inverted and confocal microscope (Olympus IX71; Olympus) (Leica TCS SP5 II; Leica, Mannheim, Germany).

Western blot analysis. Cells were washed with PBS (pH 7.4), and incubated with $2 \mathrm{X}$ concentrated electrophoresis sample buffer (125 mM Tris-HCl, pH 6.8, 5\% glycerol, 2\% SDS, 1\% $\beta$-mercaptoethanol) for $30 \mathrm{~min}$ on ice. Protein concentration was determined with Coomassie protein assay reagent using bovine serum albumin (BSA) as a standard, and equal aliquots of protein (40 mg) were separated using $8-15 \%$ SDS-PAGE. The proteins were transferred onto a nitrocellulose membrane (Millipore Corp., Billerica, MA, USA), which was blocked in TBST (TBS containing $0.1 \%$ Tween20) with 5\% non-fat dry milk for $2 \mathrm{~h}$ at room temperature and was then incubated overnight at $4{ }^{\circ} \mathrm{C}$ with the respective antibodies diluted in TBS/T/BSA (50 mM Tris- $\mathrm{HCl}, \mathrm{pH} 8.0$, $150 \mathrm{mM} \mathrm{NaCl}, 0.05 \%$ Tween-20, 0.1\% BSA). The membranes were immunoblotted with the respective antibodies and then incubated with horseradish peroxidase-conjugated secondary antibodies. Enhanced chemiluminescence (ECL) detection system (Bio-Rad Laboratories, Richmond, CA, USA) was 
used to visualize immunoreactive bands. Antibodies against Beclin-1, P62 were purchased from ProteinTech Group, Inc. (Wuhan, China), LC3B was purchased from Sigma-Aldrich (St. Louis, MO, USA), p-c-JUN and c-JUN were from Santa Cruz Biotechnology, Inc. (Santa Cruz, CA, USA). LC3B and phospho-JNK, JNK were from Cell Signaling Technology, Inc. (Beverly, MA, USA). All of the primary antibodies were used at a 1:1,000 dilution. All of the western blot analyses were replicated a minimum of three times, and densitometry comparing the proteins of interest with GAPDH as a loading control was conducted using ImageJ software.

LysoTracker Red. After washing twice with PBS, the monolayer A549 cells were labeled with $50 \mathrm{nM}$ LysoTracker Red (Beyotime Institute of Biotechnology) for $30 \mathrm{~min}$ in serum-free DMEM under $5 \% \mathrm{CO}_{2}$ at $37^{\circ} \mathrm{C}$. Images were obtained using the fluorescence microscope (Olympus).

Monodansylcadaverine (MDC) staining. Cell monolayers were cultured for $24 \mathrm{~h}$ in 2-well glass-covered chamber slides and then treated with various drugs. The slides were washed with culture medium without serum. Then, the cells were exposed to $50 \mathrm{mM}$ MDC (Nanjing KeyGen Biotech Co., Ltd.), an autofluorescent dye, for $10 \mathrm{~min}$ at $37^{\circ} \mathrm{C}$ and visualized using a confocal laser scanning microscope (Olympus IX71 and Leica TCS SP5 II). At least 3 areas per well were analysed. Two wells were analysed per treatment and per time. The experiment was repeated three times.

Transmission electron microscopy. A549 cells were fixed with $2.5 \%$ glutaraldehyde and post-fixed with $1 \%$ osmium tetroxide. After being dehydrated in increasing concentrations of alcohol, the cell pellets were embedded in epon. Representative areas were chosen for ultrathin sectioning and examined on a transmission electron microscope (JEM-2000EX; Jeol, Ltd., Tokyo, Japan).

$D C F H-D A$. Intracellular ROS generation was measured withan ROS assay with dichlorofluorescein diacetate (DCFH-DA; Beyotime Institute of Biotechnology). Briefly, the cells were plated at a density of $2 \times 10^{5}$ cells/well in six-well plates. Following treatment, intracellular ROS were detected via fluorescence microscope using DCFH-DA staining. Then, the cells were incubated with DCFH-DA $(5 \mu \mathrm{M})$ for $30 \mathrm{~min}$ at $37^{\circ} \mathrm{C}$ in the dark and were washed three times with serum-free medium. The levels of ROS were determined by Olympus BX83 fluorescence microscope (Olympus).

Statistical analysis. The data are expressed as the means \pm standard deviations (SDs) of at least 3 separate experiments. SPSS 19.0 software was used for statistical analysis. Multiple group comparisons were analysed by one-way ANOVA; 2-group comparisons were performed with Student's t-test. $\mathrm{p}<0.05$ was considered statistically significant.

\section{Results}

Fentanyl reduces the sensitivity of cisplatin in lung cancer cells. The viabilities of A549 and SPC-A1 cells concurrently treated with fentanyl and cisplatin for $24 \mathrm{~h}$ were analysed using a CCK-8 assay. As shown in Fig. 1A, fentanyl reduced the cisplatin-induced inhibition of cell viability in a concentration-dependent manner in the two cell lines, and brightfield images yielded the same result (Fig. 1B). Cisplatin-induced cell death in lung cancer cells potentially involves multiple signalling pathways. Among these pathways, apoptotic events play a critical role. To confirm this possibility, we assayed cell apoptosis by flow cytometry. The data, as indicated by the Annexin V-FITC assay, revealed that the apoptosis rates were $5.57,23.97,4.12$ and $10.23 \%$ in the control, cisplatin, fentanyl and combined groups, respectively (Fig. 1C), indicating that fentanyl attenuates the apoptosis induced by cisplatin. Nuclear condensation was investigated by DAPI staining in fentanyl and cisplatin-treated lung cancer cells. As shown in Fig. 1D, cisplatin increased the frequency of apoptotic nuclei in the A549 cell lines, and fentanyl reversed this process.

Fentanyl promotes autophagy in A549 cells. We proposed that the impact of fentanyl on cisplatin was exerted primarily through the promotion of autophagy (21). To determine whether fentanyl induces autophagy, we treated A549 cells with fentanyl at two concentrations. The conjugation of the soluble form of LC3 (LC3-I) with phosphatidylethanolamine and the conversion of LC3-I to a nonsoluble form (LC3-II) have been generally recognized as useful signs of autophagy. Thus, we examined LC3B-II expression. After treatment with fentanyl $(0.01$ or $0.1 \mu \mathrm{M})$, LC3B-II levels increased in a concentration-dependent manner (Fig. 2A). However, we could not ascertain whether the increase in LC3B-II levels was due to the activation of autophagy or the blockade of autophagosome-lysosome fusion. Thus, we subsequently measured the protein level of p62, a selective substrate of autophagy, because activation of the autophagic flux leads to p62 degradation. It is now recognized that although autophagosome formation is a necessary component of the autophagic process, formation can occur without completion of autophagy and degradation of the autophagosomal content (22). To evaluate autophagic flux, p62 protein levels were monitored by western blot analysis (21). As Fig. 2A shows, a marked decrease in p62 was detected (23). LysoTracker Red and MDC are fluorescent substances often used to detect the occurrence of autophagy. As shown in Fig. 2B, compared with the control group, fentanyl induced far more red spots and a stronger punctate fluorescence of MDC at the 24-h recovery point in the cytoplasms of A549 cells. To further evaluate the effects of fentanyl on A549 cells at the ultrastructural level, TEM was performed on the fentanyl-treated cells (8). Compared with the control group, the number of autophagic vacuoles of the fentanyl group were markedly increased. Taken together, these results demonstrate that fentanyl promotes autophagy in A549 cells (24).

ROS mediates fentanyl-induced autophagy in A549 cells. To investigate whether ROS play a role in fentanyl-induced autophagy, A549 cells were labelled using DCFH-DA, a fluorochrome that detects hydroperoxide generation. The percentage of A549 cells exhibiting increased hydroperoxide was greatly increased after one day of exposure to fentanyl compared with the control cells (Fig. 3A). Pre-incubation with NAC ( $5 \mathrm{mmol} / \mathrm{l})$, a typical antioxidant, markedly inhibited fentanyl-induced ROS generation (Fig. 3C). We then tested 

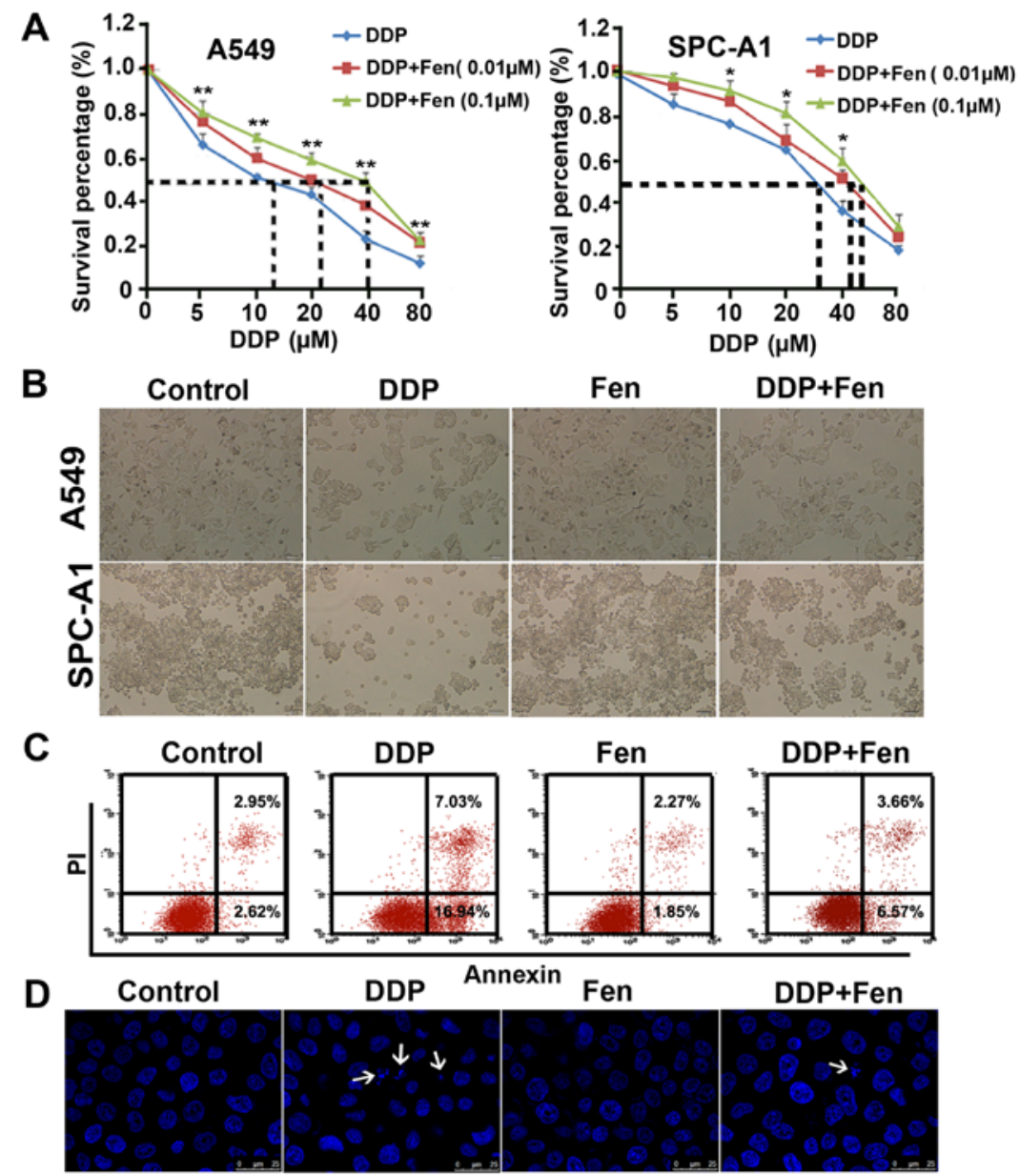

Figure 1. Fentanyl reduces the sensitivity of cisplatin in lung cancer cells. (A) A549 and SPC-A1 cells were treated with DDP at concentrations of 0,5, 10, 20, 40 and $80 \mu \mathrm{M}$ and combined with fentanyl at a concentration of 0.01 or $0.1 \mu \mathrm{M}$. The CCK-8 assay was performed. The $50 \%$ inhibitory concentration (IC F $_{50}$ ) is indicated by the dashed line. The results are presented as the means \pm standard deviations (SDs) for at least three independent experiments. ${ }^{* *} \mathrm{p}<0.01,{ }^{*} \mathrm{p}<0.05$. (B) The two cell lines were either untreated (control) or treated with fentanyl, DDP or combined for $24 \mathrm{~h}$, and brightfield images were obtained. (C) Apoptosis was measured by Annexin V/PI double-staining followed by flow cytometry analysis. (D) A549 cells were treated as in (B), followed by DAPI staining to investigate nuclear condensation. The arrow heads indicate cells with nuclear condensation.

the effect of NAC on autophagy by western blot analysis, LysoTracker Red and MDC staining. As shown in Fig. 3B, NAC inhibited fentanyl-induced autophagy, LC3BII and Beclin-1 protein levels were downregulated and P62 levels were upregulated. LysoTracker Red and MDC staining indicated similar results. Taken together, fentanyl induces ROS overproduction, which contributes to autophagy in A549 cells (23).

The ROS-activated JNK pathway contributes to fentanyl-induced autophagy. ROS are inducers or mediators of the activation of MAPK family members, including JNK, p38 and ERK1/2 (25). Additionally, studies have shown that MAPKs play a pivotal role in autophagy $(16,26,27)$. In this study, we observed that fentanyl induced JNK phosphorylation in a concentration-dependent manner (Fig. 4A). Therefore, we next asked whether fentanyl-induced autophagy involves JNK. To this end, a selective inhibitor of JNK, SP600125, was employed. As shown in Fig. 4A, pretreatment with SP600125 potently inhibited the activation of the JNK pathway and the LC3-II expression induced by fentanyl. MDC and LysoTracker Red staining yielded similar results (Fig. 4C). To investigate the role of ROS induction in fentanyl-induced autophagy and the activation of the JNK pathway more extensively, the ROS scavenger NAC was utilized. As shown in Fig. 4B, upregulation of fentanyl-induced c-Jun phosphorylation, and LC3-II expression was attenuated significantly by pretreatment with NAC. In conclusion, the ROS/JNK axis is involved in fentanyl-associated autophagy.

Inhibition of autophagy restores lung cancer cell sensitivity to cisplatin under fentanyl exposure. The data above demonstrated that fentanyl protected A549 and SPC-A1 cells from cisplatin. It has recently been reported that under adverse conditions, such as hypoxia, cancer cells make themselves adaptive by activating autophagy (9); thus, we hypothesized that autophagy may contribute to the fentanylinduced cisplatin sensitivity decline in lung cancer cells. To test this hypothesis, we inhibited autophagy using 3-MA. Cell viability was assessed by CCK-8, DAPI staining was performed to evaluate nuclear condensation, and autophagyrelated proteins were detected by western blot analysis. First, we found that pretreatment with 3-MA for $1 \mathrm{~h}$ followed by DDP treatment for $24 \mathrm{~h}$ greatly increased DDP-induced A549 cell death (Fig. 5A). DAPI had similar result (Fig. 5B). These data indicate that autophagy may act as a suppressor of A549 cells in conjunction with DDP treatment (28). Next, 

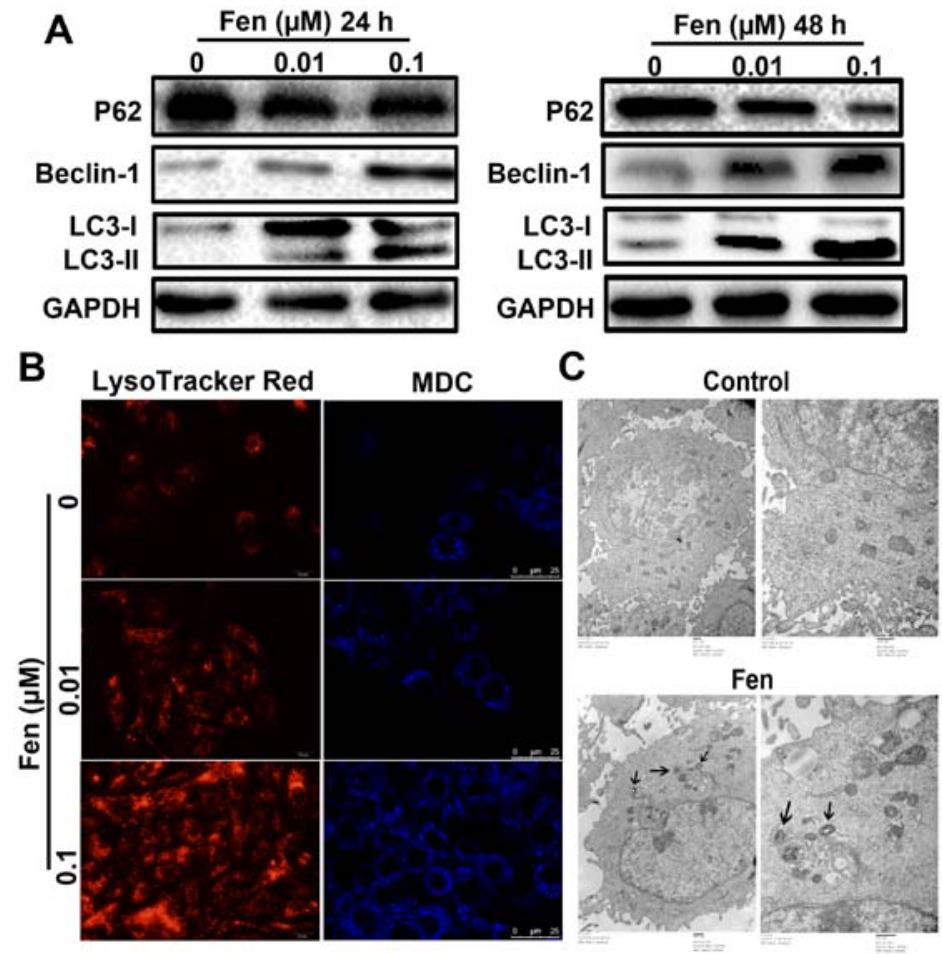

Figure 2. Promotion of autophagy by fentanyl in A549 cells. (A) Western blot analysis of the autophagy hallmark proteins P62, Beclin-1 and LC3 after incubating the A549 cells with 0.01 or $0.1 \mu \mathrm{M}$ fentanyl for 24 or $48 \mathrm{~h}$. GAPDH was utilized as a loading control. The results are presented as the means \pm standard deviations (SDs) for at least three independent experiments. (B) LysoTracker Red and MDC staining of the autophagic vesicles was performed, and images were taken using an inverted fluorescence microscope and by confocal microscopy. (C) A549 cells were treated with $0.1 \mu \mathrm{M}$ fentanyl for $24 \mathrm{~h}$ and were subjected to electron microscopy. Autophagic vacuoles are indicated by the arrows. The scale bars indicate the magnification.

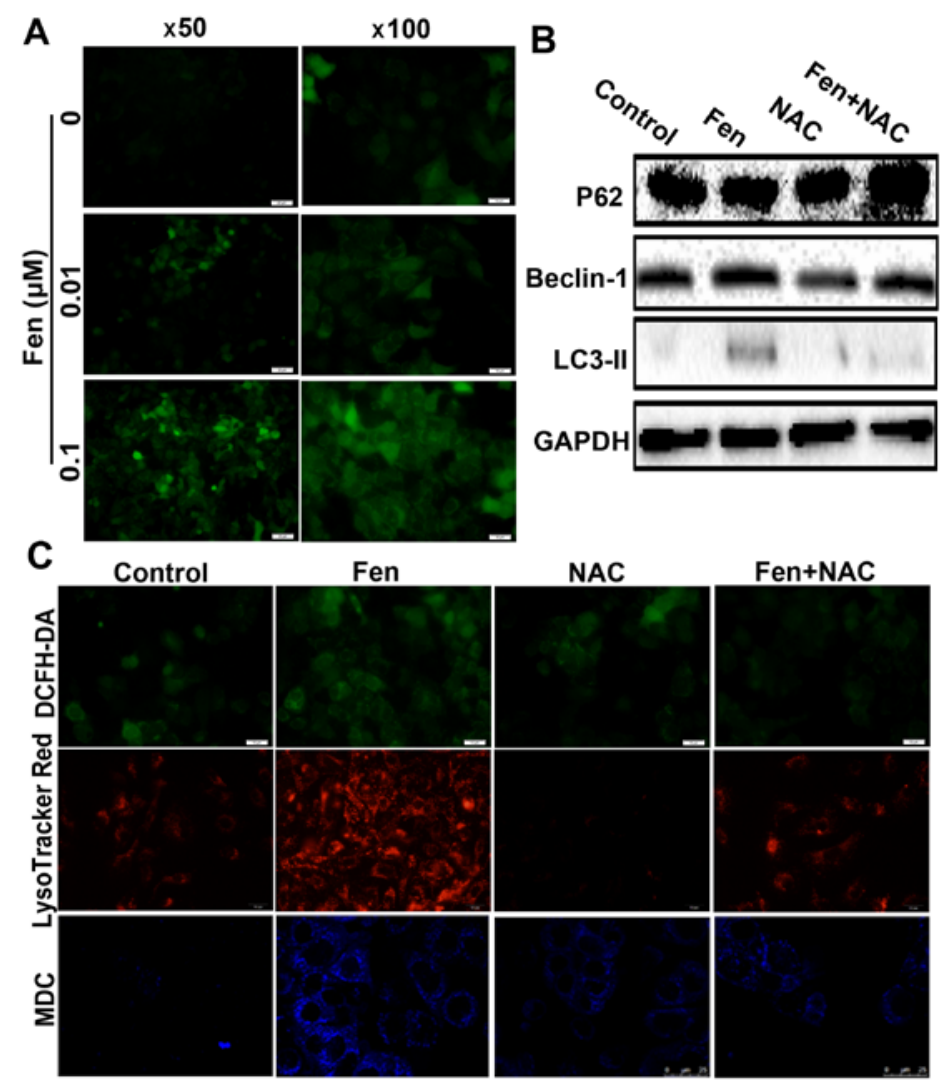

Figure 3. Reactive oxygen species (ROS) mediates fentanyl-induced autophagy in A549 cells. (A) A549 cells were treated with 0.01 or $0.1 \mu \mathrm{M}$ fentanyl for $24 \mathrm{~h}$ and then loaded with DCFH-DA for $30 \mathrm{~min}$. The levels of ROS were determined by fluorescence microscopy. (B) A549 cells were treated with $0.1 \mu \mathrm{M}$ fentanyl in the absence or presence of the antioxidant NAC $(0.5 \mathrm{mM})$ for $24 \mathrm{~h}$. The protein levels of P62, Beclin-1 and LC3B II were detected by western blot analysis, and GAPDH was utilized as a loading control. (C) The cells were treated as (B), and LysoTracker Red, MDC and DCFH-DA staining patterns were observed using an inverted fluorescence microscope and by confocal microscopy. 


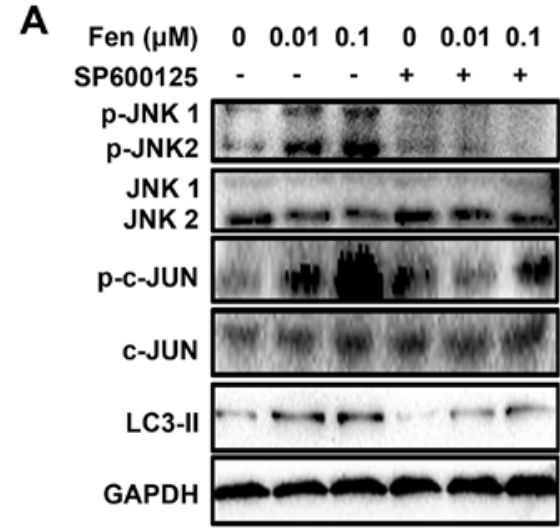

C

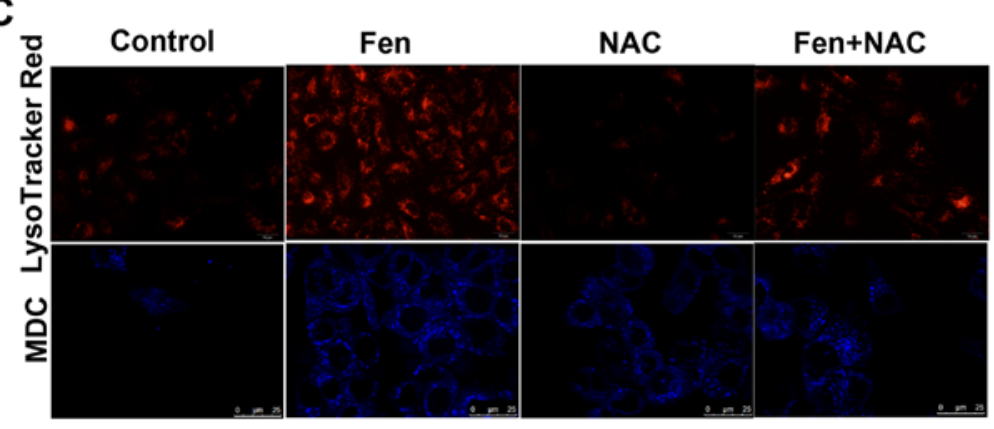

B Fen $(\mu \mathrm{M}) \quad 0 \quad 0.1 \quad 0 \quad 0.1$

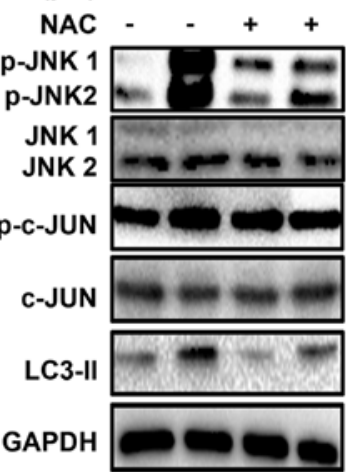

GAPDH $\mathrm{SP} 600125(20 \mu \mathrm{M})$ for $1 \mathrm{~h}$ and were then incubated with 0.01 or $0.1 \mu \mathrm{M}$ fentanyl for $24 \mathrm{~h}$. The cells were harvested and subjected to western blot analysis with the indicated antibodies. GAPDH was utilized as a loading control. (B) The cells were pretreated with NAC (5 mM) for $1 \mathrm{~h}$ and were then incubated with $0.1 \mu \mathrm{M}$ fentanyl for $24 \mathrm{~h}$. The cells were harvested and subjected to western blot analysis with the indicated antibodies. GAPDH was utilized as a loading control. (C) A549 cells were treated with SP600125 $(20 \mu \mathrm{M})$ for $1 \mathrm{~h}$ and were then incubated with $0.1 \mu \mathrm{M}$ fentanyl for $24 \mathrm{~h}$. LysoTracker Red and MDC staining was observed using an inverted fluorescence microscope and by confocal microscopy.

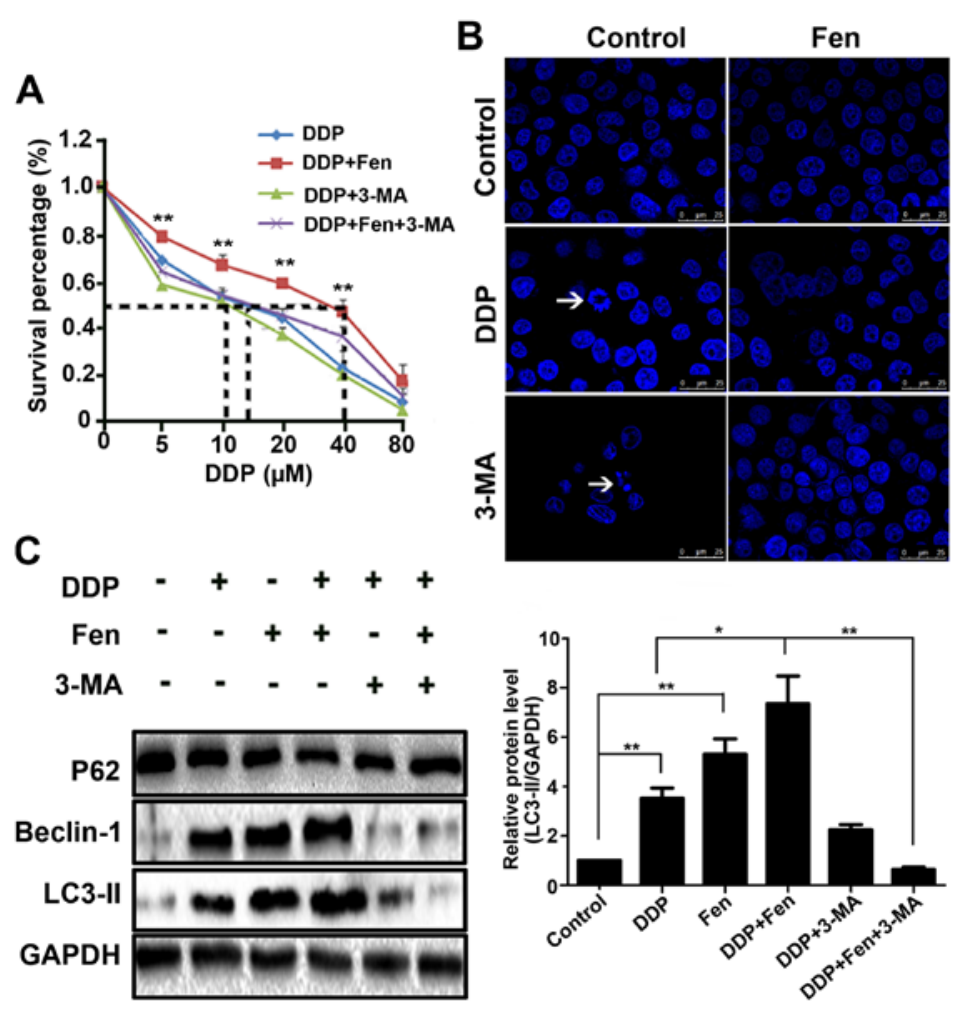

Figure 5. 3-Methyladenine (3-MA) weakens the effect of fentanyl on cisplatin in A549 cells. (A) A549 cells were treated with cisplatin at concentrations of 0, $5,10,20,40$ and $80 \mu \mathrm{M}$ for $24 \mathrm{~h}$ combined with fentanyl $(0.1 \mu \mathrm{M})$ or 3-MA, and cell viability was assessed by CCK-8. The $\mathrm{IC}_{50}$ is indicated by the dashed line. (B) DAPI staining was used to investigate nuclear condensation. The arrowheads indicate cells with nuclear condensation. (C) A549 cells were pretreated with 3-MA $(0$ or $10 \mathrm{mM})$ for $1 \mathrm{~h}$, followed by exposure to fentanyl $(0$ or $0.1 \mu \mathrm{M})$ and cisplatin $(0$ or $10 \mu \mathrm{M})$ for $24 \mathrm{~h}$. P62, Beclin-1 and LC3B II expression levels were detected by western blot analysis. GAPDH was utilized as a loading control. ImageJ software was used to quantitatively analyse the protein expression profiles observed in the western blot assay. The results are presented as the means \pm SEM for at least three independent experiments. ${ }^{* *} \mathrm{p}<0.01,{ }^{*} \mathrm{p}<0.05$. 
we found that cisplatin significantly induced the expression of LC3-II and Beclin-1 protein levels in A549 cells. Interestingly, cisplatin-induced expression levels of the above proteins were significantly increased when combined with fentanyl (Fig. 5C). These data imply that autophagy mediates the sensitivity decline of cisplatin resistance in lung cancer cells and that the inhibition of autophagy restores lung cancer cell sensitivity to cisplatin therapy.

\section{Discussion}

Cancer patients often need to use both analgesic and antitumour drugs simultaneously. As a result, research focused on how an analgesic influences the effect of an antitumour drug is necessary. However, there have been very few studies describing the role of fentanyl in the chemoresistance of cancer cells (29). Therefore, this study aimed to investigate whether fentanyl reduces the sensitivity of cisplatin in NSCLC cells.

It is believed that autophagy plays a critical role in the pathogenesis of diverse diseases, such as inflammatory bowel disease, neuronal degeneration, ageing and cancer $(28,30)$. In recent years, autophagy has become increasingly relevant in cancer research (31). One study found that the activation of autophagy induces cell death, but autophagy protects the effect of antitumour therapy by inhibiting tumour cell apoptosis (22).

Recent studies have struggled to reveal the complex paradoxical role of autophagy in cancer development and cancer therapy (32). Wu et al demonstrated that the augmented induction of autophagy by hypoxia decreased lung cancer cell susceptibility to cisplatin-induced apoptosis (9). Activated autophagy protects tumour cells from targeted therapies, including trastuzumab in breast cancer, imatinib mesylate in Philadelphia chromosome-positive cells, and proteasome inhibitors in prostate cancer (33). Li et al reported that autophagy was activated as a protective mechanism against the cellular effects of 5-fluorouracil (5-FU)-treatment and that the inhibition of autophagy by 3-MA augmented 5-FU-induced apoptosis in colon cancer cells (34). Tang et al found that glycerrhetinic acid (GA) induces cytoprotective autophagy in NSCLC cells by activating the IRE1-JNK/c-jun pathway. Autophagy induced by gefitinib in lung cancer cells is cytoprotective $(35,36)$. Furthermore, crizotinib activates autophagy in lung cancer cells (37).

However, there is no research focused on the analgesic effect of autophagy in cancer cells. Our study is the first to demonstrate that fentanyl improves the level of autophagy in A549 cells and then reduces the sensitivity to cisplatin. After treatment with cisplatin for $24 \mathrm{~h}$, the $\mathrm{IC}_{50}$ values of the A549 and SPC-A1 cells were 10 and $5 \mu \mathrm{M}$; when combined with 0.01 or $0.1 \mu \mathrm{M}$ fentanyl, the $\mathrm{IC}_{50}$ values were higher. Brightfield images demonstrated similar results. Flow cytometry and DAPI staining all indicate that fentanyl weakens cisplatin-induced apoptosis. In this study, we investigated the functional role of autophagy in A549 cells exposed to fentanyl, as evidenced by the accumulation of LysoTracker Red and MDC-positively-stained acidic vesicles, the formation of autophagosomes observed via TEM and the enhanced conversion of LC3-II and Beclin-1.

Autophagy occurs when cisplatin induces apoptosis in A549 lung cancer cells, which ultimately results in cancer cell survival. Similarly, Fig. 5 shows that cisplatin itself induced autophagy and that when the cells were pretreated with 3-MA for $1 \mathrm{~h}$ followed by DDP treatment for 24 h, DDP-induced A549 cell death was greatly increased (Fig. 5A). These data indicated that autophagy may act in a protective manner to suppress A549 cells in conjunction with cisplatin treatment. Cisplatininduced autophagy was significantly increased when combined with fentanyl, resulting in reduced sensitivity to cisplatin.

Recent investigations demonstrate that autophagic cell death is associated with alterations in ROS levels and/or the JNK signalling pathway (38). ROS are highly reactive molecules formed by incomplete one-electron reduction of oxygen, including oxygen ions and peroxides. ROS form as a natural by-product of the normal metabolism of oxygen and participate in cell signalling and homeostasis at low levels. However, under environmental stress, high levels of ROS cause irreversible oxidative damage to cellular structures (39). In this study, we found that fentanyl treatment resulted in an increase in ROS in A549 cells. The ROS inhibitor, NAC, attenuated fentanyl-induced autophagy.

Several studies report that the activation of the JNK signalling pathway by ROS represents a novel mechanism of autophagic induction $(40,41)$. In the present study, fentanyl induced significant increases in ROS generation and JNK phosphorylation. The ROS inhibitor, NAC, completely reversed the fentanyl-induced inhibition of cell proliferation, apoptosis and autophagy. In addition, the JNK inhibitor also significantly attenuated these effects. In the present study, for the first time, we demonstrate that ROS-JNK-autophagy was activated rather than suppressed in fentanyl-treated cells compared with controls, which was blocked by the inhibitors NAC and SP600125 (42).

Blocking cancer cell autophagy is emerging as a novel approach to enhancing the efficiency of chemotherapy in cancer treatment. Currently, inhibitors of autophagy that sensitize chemoresistant cells to anticancer therapy are being investigated in clinical trials $(43,44)$. As activation of hVps34/PtdInsKC3 is important for the development of the autophagosome, we evaluated whether the class III PtdIns3K inhibitor, 3-MA, inhibits autophagy and improves the cytotoxic effects of cisplatin (45). When lung cancer cells were treated with 3-MA, the cytotoxic effects of cisplatin were significantly enhanced, and the rate of apoptosis increased, which opposed the effects of fentanyl (9).

In conclusion, our study is the first to demonstrate that fentanyl reduces the sensitivity of cisplatin through the ROS-JNK-autophagy pathway. This compelling evidence expands our understanding of the benefits and clinical applications of fentanyl therapy and suggests that adding an autophagy inhibitor maybe an effective way of solving this problem.

\section{Acknowledgements}

The study was supported by the National Natural Science Foundation of China Research Grant (81273923).

\section{References}

1. Zinner R, Visseren-GrulC, Spigel DR and Obasaju C: Pemetrexed clinical studies in performance status 2 patients with non-small cell lung cancer (Review). Int J Oncol 48: 13-27, 2016. 
2. Calbó J, Meuwissen R, van Montfort E, van Tellingen O and Berns A: Genotype-phenotype relationships in a mouse model for human small-cell lung cancer. Cold Spring Harb Symp Quant Biol 70: 225-232, 2005

3. Verdecchia A, Francisci S, Brenner H, Gatta G, Micheli A, Mangone L and Kunkler I; EUROCARE-4 Working Group: Recent cancer survival in Europe: A 2000-02 period analysis of EUROCARE-4 data. Lancet Oncol 8: 784-796, 2007.

4. Ryan M, Moynihan TJ and Loprinzi CL: As-needed morphine: Yes, but at what dose and at what interval? J Clin Oncol 23 . 3849-3852, 2005.

5. Zhang XL, Chen ML and Zhou SL: Fentanyl increases colorectal carcinoma cell apoptosis by inhibition of NF- $\kappa \mathrm{B}$ in a Sirt1-dependent manner. Asian Pac J Cancer Prev 15 10015-10020, 2014.

6. Levine B and Kroemer G: Autophagy in the pathogenesis of disease. Cell 132: 27-42, 2008.

7. Wirawan E, Vanden Berghe T, Lippens S, Agostinis P and Vandenabeele P: Autophagy: For better or for worse. Cell Res 22: 43-61, 2012.

8. Ma J, Wan J, Meng J, Banerjee S, Ramakrishnan S and Roy S Methamphetamine induces autophagy as a pro-survival response against apoptotic endothelial cell death through the Kappa opioid receptor. Cell Death Dis 5: e1099, 2014

9. Wu HM,Jiang ZF, Ding PS, Shao LJ and Liu RY: Hypoxia-induced autophagy mediates cisplatin resistance in lung cancer cells. Sci Rep 5: 12291, 2015.

10. Nomura Y, Kawaraguchi Y, Sugimoto H, Furuya $H$ and Kawaguchi M: Effects of morphine and fentanyl on 5-fluorouracil sensitivity in human colon cancer HCT116 cells. J Anesth 28 : 298-301, 2014

11. Lee J, Giordano S and Zhang J: Autophagy, mitochondria and oxidative stress: Cross-talk and redox signalling. Biochem J 441: 523-540, 2012

12. Jia K, Thomas C, Akbar M, Sun Q, Adams-Huet B, Gilpin C and Levine B: Autophagy genes protect against Salmonella typhimurium infection and mediate insulin signaling-regulated pathogen resistance. Proc Natl Acad Sci USA 106: 14564-14569, 2009 .

13. Maryanovich M and Gross A: A ROS rheostat for cell fate regulation. Trends Cell Biol 23: 129-134, 2013.

14. Scherz-Shouval R and Elazar Z: Regulation of autophagy by ROS Physiology and pathology. Trends Biochem Sci 36: 30-38, 2011.

15. Sui X, Kong N, Ye L, Han W, Zhou J, Zhang Q, He C and Pan H: p38 and JNK MAPK pathways control the balance of apoptosis and autophagy in response to chemotherapeutic agents. Cancer Lett 344: 174-179, 2014

16. He W, Wang Q, Srinivasan B, Xu J, Padilla MT, Li Z, Wang X, Liu Y, Gou X, Shen HM, et al: A JNK-mediated autophagy pathway that triggers c-IAP degradation and necroptosis for anticancer chemotherapy. Oncogene 33: 3004-3013, 2014.

17. Lorin S, Pierron G, Ryan KM, Codogno $P$ and Djavaheri-Mergny M: Evidence for the interplay between JNK and p53-DRAM signalling pathways in the regulation of autophagy. Autophagy 6: 153-154, 2010.

18. Davis RJ: Signal transduction by the JNK group of MAP kinases Cell 103: 239-252, 2000

19. Wong CH, Iskandar KB, Yadav SK, Hirpara JL, Loh T and Pervaiz S: Simultaneous induction of non-canonical autophagy and apoptosis in cancer cells by ROS-dependent ERK and JNK activation. PLoS One 5: e9996, 2010.

20. Ni Z, Wang B, Dai X, Ding W, Yang T, Li X, Lewin S, Xu L, Lian J and He F: HCC cells with high levels of Bcl-2 are resistant to ABT-737 via activation of the ROS-JNK-autophagy pathway. Free Radic Biol Med 70: 194-203, 2014.

21. Bristol ML, Di X, Beckman MJ, Wilson EN, Henderson SC, Maiti A, Fan Z and Gewirtz DA: Dual functions of autophagy in the response of breast tumor cells to radiation: Cytoprotective autophagy with radiation alone and cytotoxic autophagy in radiosensitization by vitamin $\mathrm{D}_{3}$. Autophagy 8: 739-753, 2012

22. Bo Q, Ma S, Han Q, Wang FE, Li X and Zhang Y: Role of autophagy in photoreceptor cell survival and death. Crit Rev Eukaryot Gene Expr 25: 23-32, 2015.

23. Liu J, Chang F, Li F, Fu H, Wang J, Zhang S, Zhao J and Yin D: Palmitate promotes autophagy and apoptosis through ROS-dependent JNK and p38 MAPK. Biochem Biophys Res Commun 463: 262-267, 2015

24. Li J, Wang F, Xia Y, Dai W, Chen K, Li S, Liu T, Zheng Y, Wang J, Lu W, et al: Astaxanthin pretreatment attenuates hepatic ischemia reperfusion-induced apoptosis and autophagy via the ROS/MAPK pathway in mice. Mar Drugs 13: 3368-3387, 2015.
25. Kyriakis JM and Avruch J: Mammalian MAPK signal transduction pathways activated by stress and inflammation: A 10-year update. Physiol Rev 92: 689-737, 2012.

26. Cagnol S and Chambard JC: ERK and cell death: Mechanisms of ERK-induced cell death - apoptosis, autophagy and senescence. FEBS J 277: 2-21, 2010.

27. Cui Q, Tashiro S, Onodera S, Minami M and Ikejima T: Oridonin induced autophagy in human cervical carcinoma HeLa cells through Ras, JNK, and P38 regulation. J Pharmacol Sci 105: 317-325, 2007.

28. Donadelli M, Dando I, Zaniboni T, Costanzo C, Dalla Pozza E, Scupoli MT, Scarpa A, Zappavigna S, Marra M, Abbruzzese A, et al: Gemcitabine/cannabinoid combination triggers autophagy in pancreatic cancer cells through a ROS-mediated mechanism. Cell Death Dis 2: e152, 2011.

29. Zhang XL, Chen ML and Zhou SL: Fentanyl inhibits proliferation and invasion of colorectal cancer via $\beta$-catenin. Int J Clin Exp Pathol 8: 227-235, 2015.

30. Echiburú-Chau C, Alfaro-Lira S, Brown N, Salas CO, Cuellar M, Santander J, Ogalde JP and Rothhammer F: The selective cytotoxicity elicited by phytochemical extract from Senecio graveolens (Asteraceae) on breast cancer cells is enhanced by hypoxia. Int J Oncol 44: 1357-1364, 2014.

31. Thorburn A, Thamm DH and Gustafson DL: Autophagy and cancer therapy. Mol Pharmacol 85: 830-838, 2014

32. Tan Q, Wang H, Hu Y, Hu M, Li X, Aodengqimuge, Ma Y, Wei C and Song L: Src/STAT3-dependent heme oxygenase-1 induction mediates chemoresistance of breast cancer cells to doxorubicin by promoting autophagy. Cancer Sci 106: 1023-1032, 2015.

33. Vazquez-Martin A, Oliveras-Ferraros C and Menendez JA: Autophagy facilitates the development of breast cancer resistance to the anti-HER 2 monoclonal antibody trastuzumab. PLoS One 4: e6251, 2009.

34. Li J, Hou N, Faried A, Tsutsumi S and Kuwano H: Inhibition of autophagy augments 5-fluorouracil chemotherapy in human colon cancer in vitro and in vivo model. Eur J Cancer 46: 1900-1909, 2010

35. Tang ZH, Zhang LL, Li T, Lu JH, Ma DL, Leung CH, Chen XP, Jiang HL, Wang YT and Lu JJ: Glycyrrhetinic acid induces cytoprotective autophagy via the inositol-requiring enzyme $1 \alpha$-c-Jun $\mathrm{N}$-terminal kinase cascade in non-small cell lung cancer cells. Oncotarget 6: 43911-43926, 2015.

36. Sui X, Kong N, Zhu M, Wang X, Lou F, Han W and Pan H: Cotargeting EGFR and autophagy signaling: A novel therapeutic strategy for non-small-cell lung cancer. Mol Clin Oncol 2: $8-12,2014$

37. You L, Shou J, Deng D, Jiang L, Jing Z, Yao J, Li H, Xie J, Wang Z, Pan Q, et al: Crizotinib induces autophagy through inhibition of the STAT3 pathway in multiple lung cancer cell lines. Oncotarget 6: 40268-40282, 2015.

38. Kim AD, Kang KA, Kim HS, Kim DH, Choi YH, Lee SJ, Kim HS and Hyun JW: A ginseng metabolite, compound K, induces autophagy and apoptosis via generation of reactive oxygen species and activation of JNK in human colon cancer cells. Cell Death Dis 4: e750, 2013

39. Martindale JL and Holbrook NJ: Cellular response to oxidative stress: Signaling for suicide and survival. J Cell Physiol 192: $1-15,2002$.

40. Kawabe T: G2 checkpoint abrogators as anticancer drugs. Mol Cancer Ther 3: 513-519, 2004.

41. Nurse P: Universal control mechanism regulating onset of M-phase. Nature 344: 503-508, 1990.

42. Zhou H, Shen T, Shang C, Luo Y, Liu L, Yan J, Li Y and Huang S: Ciclopirox induces autophagy through reactive oxygen species-mediated activation of JNK signaling pathway. Oncotarget 5: 10140-10150, 2014.

43. Livesey KM, Tang D, Zeh HJ and Lotze MT: Autophagy inhibition in combination cancer treatment. Curr Opin Investig Drugs 10: 1269-1279, 2009

44. Amaravadi RK, Lippincott-Schwartz J, Yin XM, Weiss WA, Takebe N, Timmer W, DiPaola RS, Lotze MT and White E: Principles and current strategies for targeting autophagy for cancer treatment. Clin Cancer Res 17: 654-666, 2011.

45. O'Donovan TR, O'Sullivan GC and McKenna SL: Induction of autophagy by drug-resistant esophageal cancer cells promotes their survival and recovery following treatment with chemotherapeutics. Autophagy 7: 509-524, 2011. 